\title{
Generating sequences of Lefschetz numbers of iterates
}

\author{
Grzegorz Graff ${ }^{1}$ (D) Małgorzata Lebied $\mathbf{z}^{2}$. \\ Piotr Nowak-Przygodzki ${ }^{3}$
}

Received: 2 November 2017 / Accepted: 22 May 2018 / Published online: 9 June 2018

(C) The Author(s) 2018

\begin{abstract}
Du, Huang and Li showed in 2003 that the class of Dold-Fermat sequences coincides with the class of Newton sequences, which are defined in terms of socalled generating sequences. The sequences of Lefschetz numbers of iterates form an important subclass of Dold-Fermat (thus also Newton) sequences. In this paper we characterize generating sequences of Lefschetz numbers of iterates.
\end{abstract}

Keywords Lefschetz numbers · Newton sequences · Dold-Fermat sequences · Generating sequences

Mathematics Subject Classification Primary 11B50 - 37C25 - 55M20

Communicated by A. Constantin.

Research supported by the National Science Centre, Poland, UMO-2014/15/B/ST1/01710.

Grzegorz Graff

grzegorz.graff@pg.edu.pl

Małgorzata Lebiedź

malgosilla@gmail.com

Piotr Nowak-Przygodzki

piotrnp@wp.pl

1 Faculty of Applied Physics and Mathematics, Gdańsk University of Technology, Narutowicza 11/12, 80-233 Gdańsk, Poland

2 Institute of Mathematics, University of Gdańsk, Wita Stwosza 57, 80-952 Gdańsk, Poland

3 Sopot, Poland 


\section{Introduction}

Dold-Fermat sequences constitute a class of integer sequences satisfying some congruences [cf. (2.3)] that play important role both in number theory and dynamical systems. Let us mention that the class is called Fermat sequences by some authors [5,6], while some others name it Dold sequences (cf. for example [15]). In particular, the sequences of fixed point indices of iterates are Dold-Fermat sequences, which shows the importance of this class of sequences in periodic point theory, where the existence of such congruences often leads to valuable information about behavior of orbits and dynamics of a map near fixed or periodic points.

Another important class of sequences are Newton sequences, whose definition is based on classical Newton identities. Newton sequences are defined by (and are in a one-to-one correspondence with) the so-called generating sequences (see Definition 2.9). Du, Huang and Li showed in 2003 that in fact every Dold-Fermat sequence is a Newton sequence and conversely [5].

In this paper we establish some relations between the classes of sequences mentioned above and the Lefschetz numbers of iterates. Every sequence of Lefschetz numbers of iterates of a certain map $f$ is a Dold-Fermat sequence, thus also a Newton sequence. On the other hand, the sequences of Lefschetz numbers make a proper subset of all Dold-Fermat sequences. This fact stimulates natural question formulated by K. Wójcik during the 7th Symposium of Nonlinear Analysis (Toruń, Poland 2015): what are the generating sequences of Lefschetz numbers?

The aim of this article is to present a certain characterization of generating sequences of Lefschetz numbers of iterates.

The paper is organized in the following way. In the second section we define Lefschetz numbers, Dold-Fermat sequences and Newton sequences and show the connections between them. In Sect. 3 we find the formula for the generating sequence of the sum of two Newton sequences (Theorem 3.2) which allows us to interpret the operation of assigning a generating sequence to a given Newton sequence as a group homomorphism. In this part of the paper we also give a new, alternative proof of the fact that the classes of Dold-Fermat sequences and Newton sequences coincide (Theorems 4.3 and 4.5). Section 4 is devoted to the description of the generating sequences of $\left(\operatorname{tr} A^{n}\right)_{n}$ and $\left(-\operatorname{tr} B^{n}\right)_{n}$, where $A$ and $B$ are integral matrices, expressed in term of coefficients of its characteristic polynomials. In the final Sect. 5 we gather the results of the two previous sections and describe the possible forms of generating sequences of Lefschetz numbers under given bounds on the dimensions of the corresponding homology spaces. We also illustrate how this description can be used to verify whether a given sequence is a generating sequence of some Lefschetz numbers.

\section{Lefschetz numbers of iterates, Dold-Fermat sequences and Newton sequences}

In this section we introduce three main classes of integer sequences considered in the article. 


\subsection{Lefschetz numbers of iterates}

We start with giving the definition of Lefschetz numbers, for simplicity we confine ourselves to homology with rational coefficients.

Let us consider a finite $\mathrm{CW}$-complex $K$ of dimension $m$ with the homology groups $H_{i}(K ; \mathbb{Q})$, where $i=0,1, \ldots, m$. The groups $H_{i}(K ; \mathbb{Q})$ are finite dimensional linear spaces over $\mathbb{Q}$. For a self-map $f$ of $K$ we denote by $f_{* i}$ the linear map induced by $f$ on $H_{i}(K ; \mathbb{Q})$ and by $f_{*}$ the self-map $\bigoplus_{i=0}^{m} f_{* i}$ of $\bigoplus_{i=0}^{m} H_{i}(K ; \mathbb{Q})$. The Lefschetz number $L\left(f^{n}\right)$ of $f^{n}$ is then equal to

$$
L\left(f^{n}\right)=\sum_{i=0}^{m}(-1)^{i} \operatorname{tr}\left(f^{n}\right)_{* i}
$$

where $\operatorname{tr}\left(f^{n}\right)_{* i}$ is the trace of the integer matrix representing $\left(f^{n}\right)_{* i}: H_{i}(K ; \mathbb{Q}) \rightarrow$ $H_{i}(K ; \mathbb{Q})$. Observe that if $A$ is a matrix of $f_{* i}$, then $A^{n}$ is a matrix of $\left(f^{n}\right)_{* i}$, representing the homomorphism induced on $H_{i}(K ; \mathbb{Q})$ by $f^{n}$ (cf. [15]).

Remark 2.1 The Lefschetz number is always an integer (cf. [2]).

Let us divide homology groups into the odd-dimensional and the even-dimensional ones:

$$
H_{\mathrm{ev}}(K ; \mathbb{Q}):=\bigoplus_{i-\text { even }} H_{i}(K ; \mathbb{Q}), \quad H_{\mathrm{odd}}(K ; \mathbb{Q}):=\bigoplus_{i-\text { odd }} H_{i}(K ; \mathbb{Q})
$$

and analogously for the induced homomorphisms

$$
f_{* \mathrm{ev}}: H_{\mathrm{ev}}(K ; \mathbb{Q}) \rightarrow H_{\mathrm{ev}}(K ; \mathbb{Q}), \quad f_{* \text { odd }}: H_{\text {odd }}(K ; \mathbb{Q}) \rightarrow H_{\text {odd }}(K ; \mathbb{Q}) .
$$

Then

$$
L\left(f^{n}\right)=\operatorname{tr} f_{* \mathrm{ev}}^{n}-\operatorname{tr} f_{* \text { odd }}^{n}
$$

As a consequence, every sequence of Lefschetz numbers can be represented as the difference of traces of powers of two integer matrices, i.e.

$$
L\left(f^{n}\right)=\operatorname{tr} A_{\mathrm{ev}}^{n}-\operatorname{tr} B_{\mathrm{odd}}^{n},
$$

where $A_{\text {ev }}$ and $B_{\text {odd }}$ are the matrices representing $f_{* \text { ev }}$ and $f_{* \text { odd }}$, respectively. In the later part of the paper, we will use the formula (2.2) as the definition of the sequence of Lefschetz numbers, usually abstracting from the topological background. In fact, note that for any sequence having the form of the right-hand side of (2.2) one can find its topological realization as Lefschetz numbers.

Remark 2.2 Let $M_{i}(\mathbb{Z})$ denote the set of integer $i \times i$ matrices. Then for each pair of matrices $A_{\mathrm{ev}} \in M_{n_{1}}(\mathbb{Z}), B_{\text {odd }} \in M_{n_{2}}(\mathbb{Z})$, there exist a finite $\mathrm{CW}$-complex $K$ and a self-map $f: K \rightarrow K$ satisfying $L\left(f^{n}\right)=\operatorname{tr} A_{\mathrm{ev}}^{n}-\operatorname{tr} B_{\text {odd }}^{n}$ for every $n \in \mathbb{N}$ [15]. 
Let us remark that the sequence of Lefschetz numbers of iterates is a very useful device in periodic point theory, cf. $[4,11,12,15,18]$ and the references therein.

\subsection{Dold-Fermat sequences and their relation to Lefschetz numbers}

Definition 2.3 A sequence of integer numbers $\left(a_{n}\right)_{n=1}^{\infty}$ will be called a Dold-Fermat sequence if the following congruences (called Dold congruences or Dold relations) are fulfilled:

$$
\sum_{k \mid n} \mu(k) a_{\frac{n}{k}} \equiv 0(\bmod n) \text { for each } n \geq 1,
$$

where $\mu: \mathbb{N} \rightarrow \mathbb{Z}$ is the classical Möbius function, given by the formula

$$
\mu(n)=\left\{\begin{array}{l}
1, \quad \text { if } n=1 \\
(-1)^{k}, \quad \text { if } n=p_{1} p_{2} \ldots p_{k} \text { for different primes } p_{i} \\
0, \quad \text { otherwise. }
\end{array}\right.
$$

Remark 2.4 Consider the sequence $a_{n}=a^{n}$. For a prime number $n=p$ the congruences (2.3) take the form:

$$
a^{p}-a \equiv 0(\bmod p)
$$

which is the well-known Fermat's Little Theorem. The following generalized version of this theorem holds for traces of matrices (cf. for example Theorem 1.10 in [19]): for every $A \in M_{n}(\mathbb{Z}), p$-prime, $\alpha \in \mathbb{N}$, we have

$$
\operatorname{tr}\left(A^{p^{\alpha}}\right) \equiv \operatorname{tr}\left(A^{p^{\alpha-1}}\right)\left(\bmod p^{\alpha}\right) .
$$

Then by induction with respect to the number of primes dividing $n$ one may show that the sequence $\left(\operatorname{tr} A^{n}\right)_{n}$ satisfies the Dold congruences (Theorem 1.9 in [19]), and as a consequence any sequence of Lefschetz numbers of iterates is a Dold-Fermat sequence (for a dynamical proof cf. Lemma 10 in [5]). In fact, this statement was known in the literature much earlier and appeared in different contexts (see [22,23]).

There is a convenient way of writing down a Dold-Fermat sequence using the socalled periodic expansion, i.e. as a combination of some basic periodic sequences.

Definition 2.5 Let $k$ be a fixed natural number. We define

$$
\operatorname{reg}_{k}(n)= \begin{cases}k & \text { if } k \mid n, \\ 0 & \text { if } k \nmid n\end{cases}
$$

Thus, $\operatorname{reg}_{k}$ is the periodic sequence:

$$
(0, \ldots, 0, k, 0, \ldots, 0, k, \ldots)
$$

where the non-zero entries appear for indices divisible by $k$. 
Proposition 2.6 (cf. [19] Proposition 2.7) Any arithmetic function $\left(g_{n}\right)_{n=1}^{\infty}$ can be written uniquely in the following form of a periodic expansion:

$$
g_{n}=\sum_{k=1}^{\infty} \alpha_{k} \operatorname{reg}_{k}(n), \text { where } \alpha_{n}=\frac{1}{n} \sum_{k \mid n} \mu\left(\frac{n}{k}\right) g(k) \in \mathbb{Q} .
$$

Moreover, $g$ is integral valued and satisfies Dold congruences if and only if $\alpha_{k} \in \mathbb{Z}$ for every $k \in \mathbb{N}$.

Remark 2.7 By Proposition 2.6 every Dold-Fermat sequence is an integral combination (possibly infinite) of basic sequences $\operatorname{reg}_{k}$.

Sequences of Lefschetz numbers make a proper subset of Dold-Fermat sequences. This results from a cardinality argument: there are only countably many sequences that are given as differences of traces of powers of integer matrices, while there are uncountably many Dold-Fermat sequences. In order to show an example of a sequence that is Dold-Fermat but is not a sequence of Lefschetz numbers it is enough to observe that Lefschetz numbers cannot increase faster than exponentially.

Example 2.8 Let $g(n)=\sum_{k \mid n} k^{k}$. Then by the Möbius inversion formula $n^{n}=$ $\sum_{k \mid n} \mu(n / k) g(k)$, and thus $\alpha_{n}=\frac{1}{n} n^{n} \in \mathbb{Z}$, so $\left(g_{n}\right)_{n}$, by Proposition 2.6 , is a DoldFermat sequence. On the other hand, consider a sequence of Lefschetz numbers of iterates $\left(L\left(f^{n}\right)\right)_{n}$, which by definition has the form (2.2). Then $\left|L\left(f^{n}\right)\right| \leq c \rho^{n}$, where $c$ is some positive constant and $\rho$ is the greatest modulus of eigenvalues of matrices $A_{\mathrm{ev}}$ and $B_{\text {odd }}$ (cf. [1]). As a consequence, $\left(g_{n}\right)_{n}$ could not be a sequence of Lefschetz numbers of iterates.

Other important examples of Dold-Fermat sequences are sequences of fixed point indices of iterates. Let $f: V \rightarrow Y$ be a continuous map, where $Y$ is an Euclidean Neighborhood Retract and $V \subset Y$ is an open subset. Consider the iterates $f^{n}: V_{n} \rightarrow$ $Y$, where $V_{n}$ is defined inductively by $V_{1}=V, V_{n}=f^{-1}\left(V_{n-1}\right)$ for $n>1$, and assume that the set of fixed points of $f^{n}, \operatorname{Fix}\left(f^{n}\right)$, is compact. Then, for each $n$ the fixed point index ind $\left(f^{n}\right):=\operatorname{ind}\left(f^{n}, V_{n}\right)$ is a well-defined integer [7].

The fact that $\left(\operatorname{ind}\left(f^{n}\right)\right)_{n}$ is a Dold-Fermat sequence was proved by A. Dold in 1985 [7]. The study of fixed point indices of iterates was used in many contexts in periodic point theory and dynamics, cf. for example [3,8-11,13,21].

\subsection{Newton sequences and generating sequences}

Definition 2.9 A sequence of integer numbers $\left(a_{n}\right)_{n}$ is a Newton sequence generated by the sequence of integers $\left(c_{n}\right)_{n}$ if:

$$
a_{n}=c_{1} a_{n-1}+c_{2} a_{n-2}+\cdots+c_{n-1} a_{1}+n c_{n} .
$$

Example 2.10 The sequence $\left(a^{n}\right)_{n}$ is generated by the finite sequence $(a, 0,0, \ldots)$ while the sequence $\left(-b^{n}\right)_{n}$ is generated by the infinite sequence $\left(-b^{n}\right)_{n}$ (cf. Theorem 4.6). 
The definition of Newton sequences (cf. [14]) is based on the famous Newton identities (cf. $[6,20]$ ), which play an important role in dynamics, algebra and number theory. The class of Newton sequences encompasses many well-known types of sequences such as $k$-Lucas sequences or generalized $k$-Fibonacci sequences.

It turned out that there is a strict relation between Newton and Dold-Fermat sequences.

Theorem 2.11 (Theorem 5 and 6 in [5]) The classes of Dold-Fermat sequences and Newton sequences coincide.

Our aim is to describe the Lefschetz numbers of iterates, regarded as a proper subset of the Dold-Fermat (hence Newton) sequences in terms of their generating sequences. We want thus to answer the following question:

Problem 2.12 What are the generating sequences of sequences of Lefschetz numbers of iterates?

Note that the formula (2.6) in the definition of Newton sequence can be written as:

$$
0=-a_{n}+c_{1} a_{n-1}+c_{2} a_{n-2}+\cdots+c_{n-1} a_{1}+n c_{n} .
$$

For the sake of convenience we extend the domain of a generating sequence $\left(c_{n}\right)_{n}$ from $\mathbb{N}=\mathbb{N}^{+}$to $\mathbb{N}^{+} \cup\{0\}$, by putting $c_{0}=-1$. Then we have:

$$
0=\sum_{i=0}^{n-1} c_{i} a_{n-i}+n c_{n} .
$$

\section{The generating sequence of a sum of Newton sequences}

In this section we establish the formula for generating sequence of a sum of two Newton sequences. It turns out that this formula is almost an immediate consequence of the formal power series approach, in which the relation between a given Newton sequence and its generating sequence has very simple and elegant form.

Theorem 3.1 [Theorem 2 (1) in [6]] A sequence $\left(a_{n}\right)_{n}$ is a Dold sequence generated by $\left(c_{n}\right)_{n}$ if and only if the following formal power series equality holds:

$$
\exp \left(\sum_{n=1}^{\infty} \frac{a_{n}}{n} x^{n}\right)=\left(1-\sum_{n=1}^{\infty} c_{n} x^{n}\right)^{-1}
$$

Theorem 3.2 Assume that a sequence $\left(a_{n}\right)_{n}$ is a Newton sequence generated by a sequence $\left(c_{n}\right)_{n}$ and a sequence $\left(b_{n}\right)_{n}$ is a Newton sequence generated by a sequence $\left(d_{n}\right)_{n}$. Then the sequence $\left(a_{n}+b_{n}\right)_{n}$ is a Newton sequence generated by the sequence $\left(e_{n}\right)_{n}$ such that

$$
e_{n}=-\sum_{i=0}^{n} d_{i} c_{n-i}
$$

for $n \geq 0$. 
Proof We have: $\exp \left(\sum_{n=1}^{\infty} \frac{a_{n}+b_{n}}{n} x^{n}\right)=\exp \left(\sum_{n=1}^{\infty} \frac{a_{n}}{n} x^{n}\right) \exp \left(\sum_{n=1}^{\infty} \frac{b_{n}}{n} x^{n}\right)$.

Therefore, by Theorem 3.1

$$
1-\sum_{n=1}^{\infty} e_{n} x^{n}=\left(1-\sum_{n=1}^{\infty} c_{n} x^{n}\right)\left(1-\sum_{n=1}^{\infty} d_{n} x^{n}\right)
$$

Thus

$$
\begin{aligned}
-\sum_{n=1}^{\infty} e_{n} x^{n} & =d_{0} \sum_{n=1}^{\infty} c_{n} x^{n}+c_{0} \sum_{n=1}^{\infty} d_{n} x^{n}+\sum_{n=2}^{\infty}\left(\sum_{i=1}^{n-1} d_{i} c_{n-i}\right) x^{n} . \\
-\sum_{n=1}^{\infty} e_{n} x^{n} & =d_{0} c_{1} x+d_{1} c_{0} x+\sum_{n=2}^{\infty} d_{0} c_{n} x^{n}+\sum_{n=2}^{\infty} d_{n} c_{0} x^{n}+\sum_{n=2}^{\infty}\left(\sum_{i=1}^{n-1} d_{i} c_{n-i}\right) x^{n} . \\
\sum_{n=1}^{\infty} e_{n} x^{n} & =-\sum_{n=1}^{\infty}\left(\sum_{i=0}^{n} d_{i} c_{n-i}\right) x^{n} .
\end{aligned}
$$

We define the operation $*$ (convolution) in the set of generating sequences:

$$
(d * c)_{n}=-\sum_{i=0}^{n} d_{i} c_{n-i}
$$

(Recall that the zero term of a generating sequence is always -1 .)

Let us consider an operator $T:(N,+) \rightarrow(G, *)$, where $N$ is the set of Newton sequences equipped with the ordinary addition and $G$ is the set of generating sequences with the operation $*$, defined by assigning to each sequence in $N$ its generating sequence.

Remark 3.3 The map $T: N \rightarrow G$ is a bijection by the definition, and a homomorphism by Theorem 3.2 , we get that $(G, *)$ is a commutative group (with $(-1,0,0, \ldots)$ as the neutral element).

Our aim, which we realize in the next sections, is to describe the set $T(L)$, where $L$ denotes the set of sequences of the Lefchetz numbers of iterates.

\section{Generating sequences of the sequences of the form $\left(\operatorname{tr} A^{n}\right)_{n}$ and $\left(-\operatorname{tr} B^{n}\right)_{n}$}

Let us consider first a simpler situation in which Problem 2.12 is reduced to the case $B_{\text {odd }}=0$, i.e. to finding a generating sequence of $a_{n}=\operatorname{tr} A_{\mathrm{ev}}^{n}$. In this case the generating sequence $\left(c_{n}\right)_{n}$ turns out to be finite (i.e. for some $m \geq 1, c_{m+1}=c_{m+2}=$ 
$\cdots=0$ ) and $c_{n}$ are equal to the coefficients of the characteristic polynomial of $A_{\mathrm{ev}}$ multiplied by -1 .

In more details, let $W=x^{m}-c_{1} x^{m-1}-c_{2} x^{m-2}-\cdots-c_{m-1} x-c_{m}$ be the characteristic polynomial of the matrix $A_{\mathrm{ev}}$. Then we may consider the companion matrix $M_{m}$ of the polynomial $W$ :

$$
M_{m}=\left[\begin{array}{ccccc}
0 & 0 & \cdots & 0 & c_{m} \\
1 & 0 & \cdots & 0 & c_{m-1} \\
0 & 1 & \cdots & 0 & c_{m-2} \\
\vdots & 0 & \ddots & 0 & \vdots \\
0 & \cdots & 0 & 1 & c_{1}
\end{array}\right]
$$

Remark 4.1 (cf. Remark 4 in [24]). The sequence $\left(-1, c_{1}, c_{2}, \ldots, c_{m}, 0,0, \ldots\right)$ is a generating sequence of $\left(\operatorname{tr} M_{m}^{n}\right)_{n}$. Since $A_{\mathrm{ev}}$ and $M_{m}$ have the same characteristic polynomial, thus for $n \geq 1$,

$$
\operatorname{tr} M_{m}^{n}=\operatorname{tr} A_{\mathrm{ev}}^{n} .
$$

As a consequence, both sequences have the same generating sequence.

Let us determine the generating sequence of the sequence $a(n)=\operatorname{reg}_{k}(n)$, where $k \in \mathbb{N}$ is fixed.

Lemma 4.2 The generating sequence of $\left(\operatorname{reg}_{k}\right)_{n}$ is $\left(e_{k}\right)_{n}=(-1,0, \ldots, 1,0, \ldots)$, where the value 1 occurs at the kth place.

Proof Consider the matrix $M_{k}$ given by the formula (4.1) with $c_{1}=c_{2}=\cdots=$ $c_{k-1}=0$ and $c_{k}=1$. Then it is easy to see that $\operatorname{tr} M_{k}^{n}=\operatorname{reg}_{k}(n)$, which ends the proof by Remark 4.1.

By the use of the language of periodic expansion, basing on Theorem 3.2 and Lemma 4.2, we are able to give a short, alternative proof of Theorem 2.11 .

Theorem 4.3 Every Dold-Fermat sequence is a Newton sequence.

Proof Let $\left(g_{n}\right)$ be a Dold-Fermat sequence. To prove the theorem it is enough to show that for any fixed natural $n_{0}$ the sequence $T\left(\left(g_{n}\right)_{n=1}^{n_{0}}\right)$ takes integer values. On the other hand, by Proposition 2.6, $g(n)=\sum_{k=1}^{\infty} \alpha_{k} \operatorname{reg}_{k}(n)$, thus for each $n \leq n_{0}$ $g(n)=\sum_{k=1}^{n_{0}} \alpha_{k} \operatorname{reg}_{k}(n)$. Consequently, by Theorem 3.2

$$
\forall_{n \leq n_{0}} T\left(g_{n}\right)(n)=\alpha_{1} T\left(\operatorname{reg}_{1}\right)(n) * \ldots * \alpha_{n_{0}} T\left(\operatorname{reg}_{n_{0}}\right)(n) .
$$

As each $T\left(\left(\operatorname{reg}_{k}\right)_{n}\right)$ is an integer sequence by Lemma 4.2, their convolution is also an integer sequence, which completes the proof.

We will denote by $e_{k}^{m}$ the convolution of $m$ copies of the sequence $e_{k}$ defined in Lemma 4.2, i.e. $e_{k}^{m}=e_{k} * \ldots * e_{k}$. Below we observe that the initial part of a generating sequence coincides with the initial part of the convolution of finitely many elementary sequences $e_{k}$. 
Lemma 4.4 For any sequence $c=\left(c_{n}\right)_{n=0}^{m_{0}}$ there exists a sequence $\left(\alpha_{n}\right)_{n=0}^{m_{0}}, \alpha_{n} \in$ $\mathbb{N} \cup\{0\}$, such that the following equality holds for each $n \leq m_{0}$ :

$$
c(n)=\left(e_{1}^{\alpha_{1}} * \ldots * e_{m_{0}}^{\alpha_{m_{0}}}\right)(n) .
$$

Proof Notice first that for a given sequence $d=\left(d_{n}\right)_{n=0}^{m_{0}}$ we have, by the formula (3.4), the following equality for $n \leq m_{0}$ and a fixed $k \leq m_{0}$ :

$$
\begin{aligned}
& \left(-1, d_{1}, d_{2}, \ldots, d_{k-1}, d_{k}, \ldots, d_{m_{0}}\right) * e_{k} \\
& \quad=\left(-1, d_{1}, d_{2}, \ldots, d_{k-1}, d_{k}+1, d_{k+1}-d_{1}, d_{k+2}-d_{2}, \ldots, d_{m_{0}}-d_{m_{0}-k}\right) .
\end{aligned}
$$

Assume that a sequence $x=\left(-1, c_{1}, c_{2}, \ldots, c_{k-1}, c_{k}^{\prime}, c_{k+1}^{\prime}, \ldots, c_{m_{0}}^{\prime}\right)$ has the needed form given by the right-hand side of the formula (4.3) for any $n \leq k-1$. Consider then the sequence $y=x * e_{k}^{c_{k}-c_{k}^{\prime}}$. By the formula (4.4) it is of the form:

$$
y=\left(-1, c_{1}, c_{2}, \ldots, c_{k-1}, c_{k}^{\prime}+\left(c_{k}-c_{k}^{\prime}\right), c_{k+1}^{\prime \prime}, \ldots, c_{m_{0}}^{\prime \prime}\right) .
$$

Repeating the same procedure finitely many times, we get the Eq. (4.3).

Theorem 4.5 Every Newton sequence is a Dold-Fermat sequence.

Proof Consider a Newton sequence $\left(a_{n}\right)_{n}$ generated by the sequence

$c=\left(-1, c_{1}, \ldots, c_{m_{0}}, c_{m_{0}+1}, \ldots\right)$. It is enough to prove that for an arbitrary $m_{0}$ and all $n \leq m_{0}$ the sequence $\left(a_{n}\right)_{n=0}^{m_{0}}$ is a Dold-Fermat sequence i.e. satisfies the Dold congruences (2.3). By Lemma 4.4 we may represent $c(n)$, for $n \leq m_{0}$ and some $\alpha_{1}, \ldots, \alpha_{m_{0}}$, in the form

$$
c(n)=\left(e_{1}^{\alpha_{1}} * \ldots * e_{m_{0}}^{\alpha_{m_{0}}}\right)(n)
$$

On the other hand, the sequence $\left(e_{k}\right)_{n}$ generates $\left(\operatorname{reg}_{k}\right)_{n}$ by Lemma 4.2, thus $\left(c_{n}\right)_{n=0}^{m_{0}}$ generates $\left(\sum_{0 \leq i \leq m_{0}} \alpha_{i} \mathrm{reg}_{i}\right)_{n}$, which by Proposition 2.6 satisfies Dold congruences. This completes the proof.

While it was not difficult to find generating sequence of $a_{n}=\operatorname{tr} A_{\mathrm{ev}}^{n}$, the situation is much more complicated when we search for generating sequences of $b_{n}=-\operatorname{tr} B_{\text {odd }}^{n}$. As it is stated below, the corresponding generating sequence in this case is infinite, and its form is given in Theorem 4.8. In the remaining part of the paper we will denote $A_{\mathrm{ev}}=A$ and $B_{\text {odd }}=B$ for short.

Theorem 4.6 Let $B \in M_{i}(\mathbb{Z})$ be a matrix that is not nilpotent. Then the generating sequence $d_{n}$ of $\left(-\operatorname{tr} B^{n}\right)_{n}$ is infinite.

In the proof of the above theorem we will make use of the following algebraic result (Proposition 1 in [16]). 
Lemma 4.7 Let $G$ and $H$ be finitely generated $\mathbb{C}$-vector spaces and let $u: G \rightarrow G$ and $v: H \rightarrow H$ be linear maps. If $\operatorname{tr}\left(u^{n}\right)=\operatorname{tr}\left(v^{n}\right)$ for every $n \geq 1$, then $u$ and $v$ have the same non-zero eigenvalues counted with their multiplicities.

Proof of Theorem 4.6. If the generating sequence $d_{n}$ of $b_{n}=-\operatorname{tr} B^{n}$ were finite, then there would exist, by the formula (4.2), a matrix $M_{m}$ (where $m$ is fixed) such that $\operatorname{tr} M_{m}^{n}=b_{n}=-\operatorname{tr} B^{n}$, thus

$$
\operatorname{tr} M_{m}^{n}+\operatorname{tr} B^{n}=0 .
$$

Consider the matrix $C=M_{m} \oplus B$. Then $\operatorname{tr} C^{n}=0$, so by Lemma $4.7 C$ is nilpotent, thus also $B$ is nilpotent. We obtain a contradiction.

Theorem 4.8 Let a sequence $\left(b_{n}\right)_{n}$ be generated by $\left(d_{n}\right)_{n}$. We consider the sequence $\left(\tilde{b}_{n}\right)_{n}$ given by the formula $\tilde{b}_{n}=-b_{n}$. Let us denote by $\left(\beta_{n}\right)_{n}$ the generating sequence of $\left(\tilde{b}_{n}\right)_{n}$. Then

$$
\beta_{n}=-\sum_{\sum_{i=1}^{n} i k_{i}=n} \frac{\left(k_{1}+\cdots+k_{n}\right) !}{k_{1} ! \cdots k_{n} !} d_{1}^{k_{1}} d_{2}^{k_{2}} \cdots d_{n}^{k_{n}}
$$

where each element of the sum in (4.6) is determined by the configuration $\left(k_{1}, \ldots, k_{n}\right)$ satisfying $\sum_{i=1}^{n} i k_{i}=n$.

Proof First of all let us notice that the generating sequence of $\left(b_{n}\right)+\left(-b_{n}\right)=0$ is, by Theorem 3.2, the sequence $\left(d_{n}\right)_{n} *\left(\beta_{n}\right)_{n}$. As a consequence, the sequences $\beta_{n}$ and $d_{n}$ are related by the following recursive relation:

$$
\beta_{n}=\sum_{i=0}^{n-1} \beta_{i} d_{n-i}
$$

Now, to prove (4.6) we use induction with respect to $n$. When $n=1$ we get $\beta_{1}+d_{1}=0$, so $\beta_{1}=-d_{1}$, therefore (4.6) holds. Assume that the formula (4.6) is valid for all $i<n$, we will show that it is true also for $i=n$.

To simplify the proof we introduce the following notation.

Definition 4.9 We define below three classes of sequences that will be used in the proof.

$$
\begin{aligned}
M & =\left\{k \in(\mathbb{N} \cup\{0\})^{\mathbb{N}}: \exists_{m} \forall_{j>m} k_{j}=0\right\}, \\
A_{i} & =\left\{k \in M: \sum_{j} j k_{j}=i\right\}, \\
B_{k^{\prime}} & =\left\{k \in M: \exists_{j_{0}} \forall_{j} \quad k_{j}=\left\{\begin{array}{ll}
k_{j}^{\prime} & \text { if } j \neq j_{0}, \\
k_{j}^{\prime}-1 & \text { if } j=j_{0} .
\end{array}\right\} .\right.
\end{aligned}
$$


Definition 4.10 We also define some symbolic notation that enables us to shorten the expressions appearing in the proof. For $k \in M$ we define

$$
k !=\prod_{j}\left(k_{j}\right) !, \quad \sum k=\sum_{j} k_{j}, \quad d^{k}=\prod_{j} d_{j}^{k_{j}} .
$$

In this notation, our inductive assumption takes the form:

$$
\forall_{i<n} \quad \beta_{i}=-\sum_{k \in A_{i}} \frac{\left(\sum k\right) !}{k !} d^{k}
$$

We want to prove that the formula (4.12) is also valid for $n$. For each $i<n$ we apply in the formula (4.7) our inductive assumption and get:

$$
\beta_{n}=\sum_{i=0}^{n-1}\left(-\sum_{k \in A_{i}} \frac{\left(\sum k\right) !}{k !} d^{k} \cdot d_{n-i}\right)=-\sum_{\substack{0 \leq i \leq n-1 \\ k \in A_{i}}}\left(\frac{\left(\sum k\right) !}{k !} d^{k} \cdot d_{n-i}\right)
$$

We will rearrange the formula (4.13). Let $k \in A_{i}$ for some $0 \leq i \leq n-1$. Then we find $k^{\prime}$ such that $k \in B_{k^{\prime}}$ and $d^{k^{\prime}}=d^{k} \cdot d_{n-i}$. Since $k \in A_{i}$, obviously $k^{\prime} \in A_{n}$. It is easy to notice that there exists a bijection between pairs $(i, k)$, where $0 \leq i \leq n-1$ and $k \in A_{i}$; and pairs $\left(k^{\prime}, k\right)$, where $k^{\prime} \in A_{n}$ and $k \in B_{k^{\prime}}$.

As a result, the formula (4.13) has the form:

$$
\beta_{n}=-\sum_{\substack{k^{\prime} \in A_{n} \\ k \in B_{k^{\prime}}}} \frac{\left(\sum k\right) !}{k !} d^{k^{\prime}}
$$

Observe also that:

$$
\sum_{k \in B_{k^{\prime}}} \frac{1}{k !}=\frac{1}{k^{\prime} !} \sum k^{\prime}
$$

As a consequence, we obtain

$$
\sum_{k \in B_{k^{\prime}}} \frac{\left(\sum k\right) !}{k !}=\left(\left(\sum k^{\prime}\right)-1\right) ! \cdot \sum_{k \in B_{k^{\prime}}} \frac{1}{k !}=\frac{\left(\sum k^{\prime}\right) !}{k^{\prime} !}
$$

Finally, substituting (4.16) to (4.14), we get

$$
\beta_{n}=-\sum_{\substack{k^{\prime} \in A_{n} \\ k \in B_{k^{\prime}}}} \frac{\left(\sum k\right) !}{k !} \cdot d^{k^{\prime}}=-\sum_{k^{\prime} \in A_{n}} \frac{\left(\sum k^{\prime}\right) !}{k^{\prime} !} d^{k^{\prime}}
$$

which is our inductive claim. This completes the proof. 


\section{Generating sequences of sequences of Lefschetz numbers of iterates}

Consider a map $f: K \rightarrow K$ and assume that we have some bounds on the dimensions of the homology spaces $\operatorname{dim} H_{\mathrm{ev}}(K ; \mathbb{Q}) \leq m_{0}$ and $\operatorname{dim} H_{\text {odd }}(K ; \mathbb{Q}) \leq n_{0}$. Under these assumptions we provide the formulas for generating sequences of Lefschetz numbers in terms of the coefficients of the characteristic polynomials of the matrices $A$ and $B$ representing the homomorphisms induced by $f$ on $H_{\mathrm{ev}}(K ; \mathbb{Q})$ and $H_{\text {odd }}(K ; \mathbb{Q})$.

Theorem 5.1 Let $A \in M_{m_{0}}(\mathbb{Z}), B \in M_{n_{0}}(\mathbb{Z})$. We denote by $-c_{1}, \ldots,-c_{m_{0}}$ and $-d_{1}, \ldots,-d_{n_{0}}$ the coefficients of their characteristic polynomials, respectively. Denote $\bar{m}_{0}=\min \left\{n, m_{0}\right\}$ and $\bar{n}_{0}=\min \left\{n, n_{0}\right\}$. Then the generating sequence $\left(\gamma_{n}\right)_{n}$ of $\left(\operatorname{tr} A^{n}-\operatorname{tr} B^{n}\right)_{n}$ is given by the formula:

$$
\gamma_{n}=\sum_{j=0}^{\bar{m}_{0}} c_{j} \sum_{\sum_{i=1}^{\bar{n}_{0}} i k_{i}=n-j} \frac{\left(k_{1}+\cdots+k_{\bar{n}_{0}}\right) !}{k_{1} ! \cdot \ldots \cdot k_{\bar{n}_{0}} !} d_{1}^{k_{1}} d_{2}^{k_{2}} \cdot \ldots \cdot d_{\bar{n}_{0}}^{k_{\bar{n}_{0}}} .
$$

Proof By Remark 3.3, the generating sequence of $\left(\operatorname{tr} A^{n}-\operatorname{tr} B^{n}\right)_{n}$ is given by

$$
\begin{aligned}
& T\left(\left(\operatorname{tr} A^{n}-\operatorname{tr} B^{n}\right)_{n}\right)=T\left(\left(\operatorname{tr} A^{n}\right)_{n}\right) * T\left(\left(-\operatorname{tr} B^{n}\right)_{n}\right) \\
& \quad=\left(-1, c_{1}, c_{2}, \ldots, c_{m_{0}}, 0, \ldots\right) *\left(-1, \beta_{1}, \beta_{2}, \ldots, \beta_{n}, \ldots\right),
\end{aligned}
$$

where $\left(\beta_{n}\right)_{n}$ is the generating sequence of $\left(-\operatorname{tr} B^{n}\right)_{n}$. Applying Theorem 4.8 and taking into account that by our assumption in the formula (4.7) $d_{i}=0$ for $i>n_{0}$, we get (5.1).

Example 5.2 Assume that $n_{0}=m_{0}=1$, then the sequence $\left(\operatorname{tr} A^{n}-\operatorname{tr} B^{n}\right)_{n}$ has the form $\left(c^{n}-d^{n}\right)_{n}$, where $c, d \in \mathbb{Z}$. We calculate its generating sequence $\gamma_{n}$ :

$$
T\left(\left(c^{n}-d^{n}\right)_{n}\right)=T\left(\left(c^{n}\right)_{n}\right) * T\left(\left(-d^{n}\right)_{n}\right)=(-1, c, 0,0, \ldots) *\left(-d^{n}\right)_{n} .
$$

Thus, for $n \geq 1$ :

$$
\gamma_{n}=-\sum_{i=0}^{n} c_{i}\left(-d^{n-i}\right)=-d^{n}+c d^{n-1}=(c-d) d^{n-1} .
$$

Due to the formulas (5.1), we may verify whether a particular sequence of integers $\left(s_{n}\right)_{n}$ can be a generating sequence of some sequence of Lefschetz numbers obtained from matrices of given dimensions $m_{0}$ and $n_{0}$. Namely, consider the diophantine system of equations: $s_{n}=\gamma_{n}$ for $n=1, \ldots, m_{0}+n_{0}$ with the $m_{0}+n_{0}$ unknown variables $c_{i}$ and $d_{i}$. If this system has a solution $\tilde{c}_{1}, \ldots \tilde{c}_{m_{0}}, \tilde{d}_{1}, \ldots \tilde{d}_{n_{0}}$, we verify whether it satisfies the rest of the equations $s_{n}=\gamma_{n}$ for $n>n_{0}+m_{0}$.

Example 5.3 Let us consider $\left(s_{n}\right)_{n}$ of the form $(0,-2,-2,4, \ldots)$ and take $m_{0}=$ $2, n_{0}=1$. Comparing $s_{n}$ with $\gamma_{n}$ for $n=1,2,3$ and multiplying both sides by -1 we get 


$$
\left\{\begin{array}{l}
d_{1}-c_{1}=0, \\
d_{1}^{2}-c_{1} d_{1}-c_{2}=2, \\
d_{1}^{3}-c_{1} d_{1}^{2}-c_{2} d_{1}=2 .
\end{array}\right.
$$

It is immediate to verify that $d_{1}=1, c_{1}=1, c_{2}=-2$ is the only solution of the system (5.2). On the other hand, this set of integers does not fulfill the fourth equation $\gamma_{4}=4$, i.e.

$$
d_{1}^{4}-c_{1} d_{1}^{3}-c_{2} d_{1}^{2}=-4
$$

Hence, the sequence $\left(s_{n}\right)_{n}$ cannot be a generating sequence of the sequence of Lefschetz numbers obtained as a difference of two matrices of dimension 2 and 1 , respectively

Another important property of the generating sequence of Lefschetz numbers is the fact that it grows not faster than exponentially (like the Lefschetz numbers themselves, cf. Example 2.8). Namely we have the following estimate (we apply the notation from Theorem 5.1):

Theorem 5.4 Assume $L\left(f^{n}\right)=\operatorname{tr} A^{n}-\operatorname{tr} B^{n}$, where $A \in M_{m_{0}}(\mathbb{Z}), B \in M_{n_{0}}(\mathbb{Z})$. Let us take the smallest constant $\sigma>0$ such that for all $j$

$$
\sqrt[j]{\left|c_{j}\right|} \leq \sigma \text { and } \sqrt[j]{\left|d_{j}\right|} \leq \sigma
$$

Then:

$$
\left|\gamma_{n}\right| \leq 2 \cdot(2 \sigma)^{n}
$$

Proof Applying the formulas (5.1) and (5.3) we get for $n>\max \left(m_{0}, n_{0}\right)$ :

$$
\begin{aligned}
\left|\gamma_{n}\right| & \leq \sum_{j=0}^{m_{0}}\left|c_{j}\right| \sum_{\sum_{i=1}^{n_{0}} i k_{i}=n-j} \frac{\left(k_{1}+\cdots+k_{n_{0}}\right) !}{k_{1} ! \cdot \ldots \cdot k_{n_{0}} !}\left|d_{1}\right|^{k_{1}}\left|d_{2}\right|^{k_{2}} \cdot \ldots \cdot\left|d_{n_{0}}\right|^{k_{n_{0}}} \\
& \leq \sum_{j=0}^{m_{0}} \sigma^{j} \cdot \sigma^{n-j} \sum_{\sum_{i=1}^{n_{0}} i k_{i}=n-j} \frac{\left(k_{1}+\cdots+k_{n_{0}}\right) !}{k_{1} ! \cdot \ldots \cdot k_{n_{0}} !} \\
& \leq \sigma^{n} \sum_{j=0}^{m_{0}} \sum_{\sum_{i=1}^{n_{0}} i k_{i}=n-j} \frac{\left(k_{1}+\cdots+k_{n_{0}}\right) !}{k_{1} ! \cdot \ldots \cdot k_{n_{0}} !} .
\end{aligned}
$$

On the other hand, by Remark 8 in [5], the following equality holds:

$$
\sum_{\sum_{i=1}^{n} i k_{i}=n} \frac{n}{\left(k_{1}+\cdots+k_{n}\right)} \frac{\left(k_{1}+\cdots+k_{n}\right) !}{k_{1} ! \cdot \ldots \cdot k_{n} !}=2^{n}-1
$$


As a consequence of (5.4), we obtain:

$$
\left|\gamma_{n}\right| \leq \sigma^{n} \cdot \sum_{j=0}^{m_{0}} 2^{n-j} \leq \sigma^{n} \cdot 2^{n+1}
$$

which is the desired estimate.

To conclude, we formulate an open problem related to the representation of Lefschetz numbers in the form of periodic expansion. By Proposition 2.6, we may represent any sequence of Lefschetz numbers as a combination of basic sequences $\operatorname{reg}_{k}$ with some coefficients $\alpha_{k}$ (called Dold coefficients), i.e.

$$
L\left(f^{n}\right)=\sum_{k=1}^{\infty} \alpha_{k} \operatorname{reg}_{k}(n) .
$$

Problem 5.5 Characterize the sequences of Dold coefficients of periodic expansion for Lefschetz numbers.

The representation of Lefschetz numbers by Dold coefficients is much different from the representation by the use of generating sequences. Notice for example that the generating sequence of $\left(\operatorname{tr} A^{n}\right)_{n}$ is finite, while the sequence of Dold coefficients of its periodic expansion may be infinite. This holds even in the simplest case for $1 \times 1$ matrix with the entry equal to 2 . In such a case the coefficients $\alpha_{k}$ of periodic expansion of the sequence $L\left(f^{n}\right)=2^{n}$ are non-zero for each $k$ (cf. [17] Theorem 1.2).

Acknowledgements We would like to thank Dr. Jakub Byszewski for valuable remarks and comments that help us to improve the paper.

Open Access This article is distributed under the terms of the Creative Commons Attribution 4.0 International License (http://creativecommons.org/licenses/by/4.0/), which permits unrestricted use, distribution, and reproduction in any medium, provided you give appropriate credit to the original author(s) and the source, provide a link to the Creative Commons license, and indicate if changes were made.

\section{References}

1. Babenko, I.K., Bogatyi, S.A.: The behavior of the index of periodic points under iterations of a mapping. Math. USSR Izv. 38, 1-26 (1992)

2. Brown, R.F.: The Lefschetz fixed point theorem. Glenview, New York (1971)

3. Chow, S.N., Mallet-Parret, J., Yorke, J.A.: A Periodic Point Index Which is a Bifurcation Invariant. Geometric Dynamics (Rio de Janeiro, 1981), 109-131. Springer Lecture Notes in Math, Berlin (1983)

4. Duan, H.-B.: The Lefschetz numbers of iterated maps. Topol. Appl. 67(1), 71-79 (1995)

5. Du, B.-S., Huang, S.-S., Li, M.-C.: Generalized Fermat, double Fermat and Newton sequences. J. Number Theory 98, 172-183 (2003)

6. Du, B.-S., Huang S.-S., Li, M.-C.: Newton, Fermat, and exactly realizable sequences. J. Integer Seq. 8, Article 05.1.2 (2005)

7. Dold, A.: Fixed point indices of iterated maps. Invent. Math. 74, 419-435 (1985)

8. Graff, G.: Minimal periods of maps of rational exterior spaces. Fund. Math. 163(2), 99-115 (2000) 
9. Graff, G.: Minimal number of periodic points for smooth self-maps of two-holed 3-dimensional closed ball. Topol. Method Nonlinear Anal 33(1), 121-130 (2009)

10. Graff, G., Jezierski, J., Nowak-Przygodzki, P.: Fixed point indices of iterated smooth maps in arbitrary dimension. J. Differ. Equ. 251, 1526-1548 (2011)

11. Graff, G., Jezierski, J.: Minimal number of periodic points of smooth boundary-preserving self-maps of simply-connected manifolds. Geom. Dedicata 187, 241-258 (2017)

12. Guirao, G., Llibre, J.: Periodic structure of transversal maps on $\mathbb{C} P^{n}, \mathbb{H} P^{n}$ and $\mathbb{S}^{p} \times \mathbb{S}^{q}$. Qual. Theory Dyn. Syst. 12(2), 417-425 (2013)

13. Hernández-Corbato, L., Le Calvez, P., Ruiz del Portal, F.: About the homological discrete Conley index of isolated invariant acyclic continua. Geom. Topol. 17(5), 2977-3026 (2013)

14. Horn, R.A., Johnson, C.A.: Matrix Analysis. Cambridge University Press, Cambridge (1990)

15. Jezierski, J., Marzantowicz, W.: Homotopy methods in topological fixed and periodic points theory. In: Topological Fixed Point Theory and Its Applications, vol. 3. Springer, Dordrecht (2006)

16. Le Calvez, P., Ruiz del Portal, F., Salazar, J.: Indices of the iterates of $\mathbb{R}^{3}$-homeomorphisms at fixed points which are isolated invariant sets. J. Lond. Math. Soc. (2) 82(3), 683-696 (2010)

17. Llibre, J., Paranõs, J., Rodriguez, J.A.: Periods for transversal maps on compact manifolds with a given homology. Houston J. Math. 24(3), 397-407 (1998)

18. Llibre, J., Todd, M.: Periods, Lefschetz numbers and entropy for a class of maps on a bouquet of circles. J. Differ. Equ. Appl. 11(12), 1049-1069 (2005)

19. Marzantowicz, W., Nowak-Przygodzki, P.: Finding periodic points of a map by use of a $k$-adic expansion. Discrete Contin. Dynam. Systems 5(3), 495-514 (1999)

20. Mead, D.G.: Newton's identities. Am. Math. Mon. 99, 749-751 (1992)

21. Pokrovskii, A., Rasskazov, O.: On the use of the topological degree theory in broken orbits analysis. Proc. Am. Math. Soc. 132(2), 567-577 (2004)

22. Steinlein, H.: 70 years of asymptotic fixed point theory. J. Fixed Point Theory Appl. 17(1), 3-21 (2015)

23. Steinlein, H.: Fermat's little theorem and Gauss congruence: matrix versions and cyclic permutations. Am. Math. Mon. 124(6), 548-553 (2017)

24. Wójcik, K.: Binomial Transform and Dold sequences. J. Integer Seq. 18(1), Article 15.1 .1 (2015) 